\title{
Improved distribution and reduced toxicity of adriamycin bound to albumin-heparin microspheres
}

\author{
H.F.M. Cremers ${ }^{\text {a }}$, R. Verrijk ${ }^{b}$, L.G. Bayon ${ }^{c}$, M.M. Wesseling ${ }^{b}$, J. Wondergem ${ }^{d}$, \\ G. Heuff ${ }^{c}$, S. Meijer ${ }^{c}$, G.S. Kwon ${ }^{\text {e }}$, Y.H. Bae ${ }^{\mathrm{e}}$, S.W. Kim ${ }^{\mathrm{e}}$, J. Feijen ${ }^{\mathrm{a}, *}$ \\ a Department of Chemical Technology, University of Twente, P.O. Box 217, 7500 AE Enschede, The Netherlands \\ ${ }^{\mathrm{b}}$ Division of Experimental Therapy, the Netherlands Cancer Institute, Plesmanlaan 121, 1066 CX Amsterdam, The Netherlands \\ ${ }^{\mathrm{c}}$ Department of Surgical Oncology, Free University Hospital, Amsterdam, The Netherlands \\ d Department of Clinical Oncology, University Hospital Leiden, Leiden, The Netherlands \\ ' Department of Pharmaceutics and Center for Controlled Chemical Delivery, University of Utah, 421 Wakaraway, Suite 318, Salt Lake \\ City, UT 84108, USA
}

Received 12 July 1994; revised 29 November 1994; accepted 5 December 1994

\begin{abstract}
Adriamycin (ADR) was formulated in albumin-heparin conjugate microspheres (AHCMS) to improve site-specific delivery and to reduce the toxicity of the drug. The effect of formulating ADR in AHCMS was investigated upon intrahepatic administration to male Wag/Rij rats. After intraveno-portal (i.v.p.) administration of ADR-AHCMS, ADR peak plasma concentrations were reduced 10-fold and ADR tissue levels of non-target tissues were significantly reduced, as compared to i.v.p. administration of the free drug. At an i.v.p. administered drug dose of 7.5 $\mathrm{mg} / \mathrm{kg}$, free ADR showed distinct signs of acute toxicity. At the same dose of ADR-AHCMS, signs of toxicity were absent. Cardiac function parameters which were determined using an isolated working heart model did not change as a result of i.v.p. administration of free ADR or ADR-AHCMS at a dose of $7.5 \mathrm{mg} / \mathrm{kg}$. Heart weights of animals in the ADR-AHCMS or the free ADR groups, however, were significantly lower than controls. Dose tolerance studies after intrahepatic-arterial (i.h.a.) administration of free ADR, empty AHCMS and ADR-AHCMS in rats demonstrated that empty AHCMS are tolerated at a dose of $45 \mathrm{mg} / \mathrm{kg}$. Free ADR was tolerated at a dose of 4 $\mathrm{mg} / \mathrm{kg}$, whereas ADR-AHCMS were tolerated up to a dose of $10 \mathrm{mg}$ ADR $/ \mathrm{kg}$, as indicated by the survival.
\end{abstract}

Keywords: Microsphere; Adriamycin; Distribution; Cardiotoxicity; Drug targeting; Liver metastasis

\section{Introduction}

Adriamycin (ADR) is considered to be one of the most effective antineoplastic agents currently

\footnotetext{
* Corresponding author. Tel. +31-53-892976; Fax +31-53317202.
}

used in cancer chemotherapy. The major drawback in ADR chemotherapy is the toxicity of the drug. Anthracyclines like ADR carry the risk of potentially lethal cardiomyopathy, characterized clinically by biventricular, progressive congestive heart failure. The amount of myocardial damage is related to the cumulative dose, and at a standard treatment of three weekly intravenous bolus 
injections, the cumulative dose should not exceed $450-550 \mathrm{mg} / \mathrm{m}^{2}$ (Bielack et al., 1989). This maximum allowable dose, however, is related to the dose schedule. Storm et al. (1989) found that reduction of cardiotoxicity induced by either continuous infusion or liposomal encapsulation of ADR is most likely related to the reduction of peak plasma levels. Moreover, it has been reported that damage to heart muscle cells is mainly related to peak ADR concentrations and that at lower concentrations higher cumulative doses are tolerated (Bielack et al., 1989). In order to increase ADR dosages while minimising undesirable side effects albumin-heparin conjugate microspheres (AHCMS) were developed as biodegradable ion-exchange ADR carriers (Cremers et al., 1994a,b,c). Site-specific administration of ADR should decrease peak plasma concentrations and thus decrease the exposure of healthy tissues to ADR. In this paper toxicity and pharmacokinetic studies are described which were performed using intraveno-portal (i.v.p.) administration of ADR to male Wag/Rij rats, either as a free drug or formulated in AHCMS. In addition, cardiotoxicity of free ADR and ADR-AHCMS after i.v.p. administration to rats was studied using an isolated working-heart model (Wondergem et al., 1991). Finally, dose tolerance of free ADR and ADR-AHCMS upon intrahepatic administration via the hepatic artery (i.h.a.) was studied in healthy Wag/Rij rats.

\section{Materials and methods}

\subsection{Materials}

Albumin-heparin conjugate microspheres (AHCMS) with a diameter of 10-25 $\mu \mathrm{m}$ (dried) were prepared by crosslinking with $0.5 \%$ glutaraldehyde as described previously (Cremers et al., 1994a). Adriamycin $\cdot \mathrm{HCl}$ was a kind gift from Pharmachemie, Haarlem, The Netherlands. Adriamycin-loaded microspheres (ADR-AHCMS) were obtained by loading AHCMS with ADR up to a payload of $25.4 \% \mathrm{w} / \mathrm{w}$ as described previously (Cremers et al., 1994c).

\subsection{Animals}

Male Wag/Rij rats, 8-12 weeks old and weighing $260 \pm 40 \mathrm{~g}$ at the time of the experiments, were bred at the animal department of The Netherlands Cancer Institute (Amsterdam, The Netherlands) under specific pathogen-free (SPF) conditions or obtained from the Centraal Proefdieren Bedrijf (T.N.O., Rijswijk, The Netherlands). The animals were kept on a $12 \mathrm{~h}$ light-12 $\mathrm{h}$ dark cycle and fed standard rat chow and plain tap water ad libitum.

\subsection{Microsphere suspensions}

ADR-AHCMS were resuspended (using a minimal amount of ethanol for wetting of the microspheres) in $5 \% \mathrm{w} / \mathrm{v}$ glucose solution. After $2 \mathrm{~h}$ of rotation at $4^{\circ} \mathrm{C}$ in the dark, the microspheres were centrifuged and the supernatant was decanted. The ADR release in this washing solution was measured by $\mathrm{UV} / \mathrm{Vis}$ spectroscopy at $A_{494}$ using a Uvikon 930 spectrophotometer (Kontron Instruments, Rotkreuz, Switzerland). Fresh glucose solution was added to the microspheres to give the final concentration needed in the experiments.

2.4. ADR-plasma levels and tissue distributions after i.v.p. administration of free $A D R$ and $A D R$ AHCMS in rats

\subsubsection{Surgical procedure of i.v.p. administration}

Under ether anaesthesia, a midline laparotomy was performed and a loop of the small intestine was exposed. Free ADR or ADR-AHCMS (both corresponding to $5 \mathrm{mg} \mathrm{ADR} / \mathrm{kg}$ ), dissolved or resuspended in $0.5 \mathrm{ml} 5 \% \mathrm{w} / \mathrm{v}$ glucose solution, was slowly injected into a mesenteric vein under microscopic observation using a $\mathbf{3 0}$ gauge needle. Afterwards, the vein was ligated. To increase the efficiency of the injection in the case of ADRAHCMS, the syringe used was refilled with $0.5 \mathrm{ml}$ of $5 \% \mathrm{w} / \mathrm{v}$ glucose solution and this was injected into another mesenteric vein. The amount of ADR injected was determined from the remainder of ADR-AHCMS in the syringe using HPLC analysis, carried out as described below. 


\subsubsection{Plasma pharmacokinetics and tissue distribu- tion}

Rats were anaesthetized using ether and a catheter was introduced into the ascending aorta via carotid artery cannulation. Blood samples were drawn at $t=0,5,15,30,60,120$ and 240 min after administration of free ADR or ADRAHCMS. Blood samples were stored at $-20^{\circ} \mathrm{C}$ until determination of the ADR content, which was performed as described below. To determine the ADR tissue distributions, rats were killed at 15,60 and $240 \mathrm{~min}$ after administration of the drug. Blood samples were taken and lung, heart, intestine, spleen, kidney and liver were removed and stored at $-20^{\circ} \mathrm{C}$ until ADR extraction and determination of the ADR content, which were performed as described below.

\subsubsection{Adriamycin tissue extraction}

Approx. $1 \mathrm{~g}$ of tissue was weighed into a $50 \mathrm{ml}$ Falcon ${ }^{\circledR}$ tube. After addition of $1 \mathrm{ml}$ of distilled water and $10 \mu \mathrm{l}$ internal standard (IS; 4-deoxydoxorubicinol) solution, the tissue was homogenized. Then, $2 \mathrm{ml}$ of borate buffer $(2.5 \mathrm{M}, \mathrm{pH}$ 9.2) was added. Organic phase $(7 \mathrm{ml}$ of $\mathrm{CHCl}_{3} / \mathrm{CH}_{3} \mathrm{OH}, 4: 1$ ), was added and the mixture was vortexed for $1 \mathrm{~min}$. After centrifugation (10 $\mathrm{min}, 4000 \mathrm{rpm}$ ) the aqueous phase was removed. The remaining organic phase which contained IS, ADR and ADR metabolites was transferred to silanized glass vials. The solvent was evaporated at $50^{\circ} \mathrm{C}$ under nitrogen flow and the remainder was redissolved in $200 \mu \mathrm{l}$ of the HPLC mobile phase. Before injection in the HPLC system the solution was centrifuged for $20 \mathrm{~min}$ at $13000 \mathrm{rpm}$. A calibration curve was determined from known amounts of ADR and IS that had been added to tissue samples from untreated rats, prior to homogenization. Calibration samples were further handled in the same way as the other samples.

\subsubsection{HPLC analysis of ADR samples}

ADR concentrations of the blood samples and the tissue extracts were determined using HPLC according to a standard procedure that was described previously (Cremers et al., 1994c). ADR tissue levels were calculated from the calibration curve using the log ratio of the peak heights of ADR and IS. Various metabolites were detected, but they did not interfere with ADR or IS.

\section{5. $A D R$ and $A D R-A H C M S$ cardiotoxicity in iso- lated working rat heart model}

Animals were given ADR or ADR-AHCMS i.v.p. as described above in three groups of 15 rats each. Rats of the first group were treated i.v.p. with $7.5 \mathrm{mg} / \mathrm{kg}$ of free ADR. Animals in the second group were administered i.v.p. ADRAHCMS, corresponding to a dose of $7.5 \mathrm{mg}$ of $\mathrm{ADR} / \mathrm{kg}$. The third group received no treatment and served as a control. After administration of ADR or ADR-AHCMS, rats were weighed weekly and blood samples were drawn from the tail vein every week. Haematocrit, haemoglobin content, leucocyte count, and urea and creatinine levels were determined as parameters for systemic toxicity. After 8 weeks, the animals were killed as described below. Heart, kidneys and liver were removed and after the perfusion experiment, histological slides were made. For light microscopic evaluation, tissues of heart and kidney were fixed in $4 \%$ formaldehyde and embedded in paraffin Paraclean $^{\otimes}$ and sections of 5-6 $\mu \mathrm{m}$ were made and stained with hematoxylin and eosin $(\mathrm{H} / \mathrm{E})$.

The heart was isolated as described by Wondergem et al. (1991). When the heart had been cooled for 30-60 s and dissected, it was lifted from the cold buffer with a blunt forceps and the aortic stump was fixed to the aortic cannula of the perfusion apparatus and after allowing the heart to recover, cardiac function was tested as described by Wondergem et al. (1991).

\subsection{Dose tolerance of ADR-AHCMS after i.h.a. administration in rats}

At day 0 , male $\mathrm{Wag} / \mathrm{Rij}$ rats were administered increasing doses of free ADR $(5,7.5,10$ $\mathrm{mg} / \mathrm{kg}, n=3$ per group) or ADR-AHCMS (5, $7.5,10,15 \mathrm{mg} \mathrm{ADR} / \mathrm{kg}, n=3$ per group) via the hepatic artery as described below. 12 rats (four groups, six rats each) were administered a dose of empty AHCMS, comparable with the ADRAHCMS doses, to determine the influence of the 
embolization effect. Furthermore, two rats served as a control and received a sham operation and were administered $5 \% \mathrm{w} / \mathrm{v}$ glucose solution. Survival of the animals was followed up to 28 days and the body weights were monitored at day 0,4 , $7,11,14,17,22$ and 25. Nephrotoxicity and hepatic toxicity were determined at day 14 by measurement of creatinine and urea levels, and $\gamma$-glutamyl transferase $(\gamma-\mathrm{GT})$, serum glutamate oxaloacetate transaminase (SGOT), serum glutamate pyruvate transaminase (SGPT) and alkaline phosphatase (AF) levels, respectively.

\subsubsection{Surgical procedure of i.h.a. administration}

Animals were anaesthetized using $1 \mu \mathrm{l} / \mathrm{g}$ body weight of a mixture of $0.2 \mathrm{ml} 2 \% \mathrm{w} / \mathrm{v}$ thiazine solution (Rompun ${ }^{\star}$, Bayer, Germany) and $0.8 \mathrm{ml}$ $10 \% \mathrm{w} / \mathrm{v}$ ketamine solution (Aescoket ${ }^{\circledR}$, Aesculaap BV, The Netherlands), and an upper middle laparotomy was performed. Under microscopic observation, the common bile duct was dissected and a vessel loop was placed around it to be used as a tractor. Subsequently, the hepatic artery was dissected from the celiac trunk up to the confluence with the gastroduodenal artery. Then the gastroduodenal artery was isolated over $0.5 \mathrm{~cm}$ and a proximal and a distal $4 / 0$ silk loop was placed. The distal one was tightened and the proximal one was pulled up in order to stretch the artery and to avoid bleeding when the artery was opened. A partial cut of the lumen of the artery was performed using microscissors and a small catheter ( 2 French gauge) was inserted into the gastroduodenal artery and pushed up to the hepatic artery. The catheter was maintained in its place by soft pressure with dissection forceps, meanwhile the infusion of ADR solution or empty or ADR loaded AHCMS suspension in $5 \% \mathrm{w} / \mathrm{v}$ glucose solution was carried out. After the infusion, the catheter was removed, the proximal ligature was tightened and the abdominal wound was closed.

\section{Results and discussion}

AHCMS and ADR-AHCMS were prepared according to standard procedures previously de- scribed by Cremers et al. (1994a,c). ADR release from ADR-AHCMS during the washing step in the suspension procedure of the microspheres in aqueous medium was as described previously (Cremers et al., 1994c). The amount of drug released in this washing step varied between 2 and $30 \% \mathrm{w} / \mathrm{w}$ of the incorporated drug depending on the batch of ADR-AHCMS used. This was taken into account in the preparation of the injections.

\subsection{ADR plasma levels and tissue distributions} after i.v.p. administration of free $A D R$ and $A D R$ AHCMS in rats

To determine the drug targeting and controlled release efficiency of ADR-AHCMS, ADR plasma levels and tissue distributions were measured after i.v.p. injection of free drug and microsphere-formulated drug. ADR clearance from blood after administration of $5 \mathrm{mg} \mathrm{ADR} / \mathrm{kg}$, either as free drug or as ADR-AHCMS, is shown in Fig. 1.

In the free ADR group the concentration of ADR in blood decreased rapidly and remained nearly constant at a low level. The initial rapid decrease in ADR concentration results from the distribution of ADR over tissues. This phase is followed by a slower elimination phase as a con-

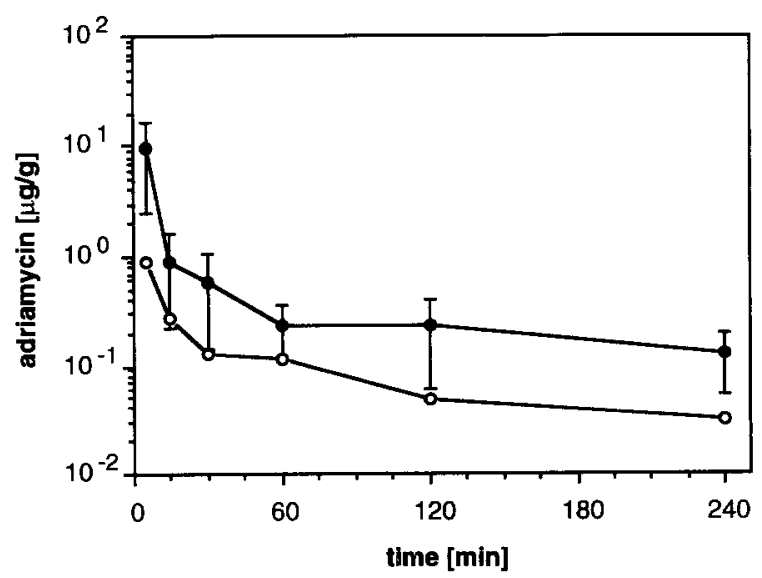

Fig. 1. Mean ADR concentrations in blood of Wag/Rij rats after i.v.p. administration of $5 \mathrm{mg} \mathrm{ADR} / \mathrm{kg}$. (•) Free ADR $(n=6)$; (O) ADR-AHCMS $(n=4)$. Error bars indicate SD. 
sequence of renal clearance and metabolism. This was also found by Camaggi et al. (1988). If the drug was administered as ADR-AHCMS, a comparable profile was observed, although the drug peak levels at $t=5 \mathrm{~min}$ were about one-tenth of that after administration of free ADR. Overall, the blood ADR concentration of free ADR was higher than that of ADR-AHCMS, and although the same dose was given, the area under the curve (AUC) of ADR-blood is smaller in the case of ADR-AHCMS as compared with free ADR $\left(\mathrm{AUC}_{\text {free }} \mathrm{ADR}>\mathrm{AUC}_{\mathrm{ADR} \text {-AHCMS }}\right)$. This may be due to more extensive metabolism of ADR in the liver when administered as ADR-AHCMS. Intraveno-portal (i.v.p.) administration of ADRAHCMS causes vascular occlusion whereupon ADR can be released locally (Cremers et al., 1994b). Therefore, more ADR is present in the liver and may be metabolized more extensively. Indications for this were observed in HPLC chromatograms of liver tissue extracts (data not shown). The formation of metabolites by bioreduction of ADR was previously reported by Willmott et al. (1990). These metabolites, however, were not analysed in this study. As already mentioned above, peak plasma levels were 10-times lower if ADR was given i.v.p. as ADR-AHCMS instead of free drug. It is therefore concluded that the use of ADR-AHCMS could prevent adverse effects related to high peak plasma levels of the drug.

The ADR tissue distributions after i.v.p. administration of ADR and ADR-AHCMS at a dose of $5 \mathrm{mg} \mathrm{ADR} / \mathrm{kg}$ are shown in Fig. 2. The tissue levels were corrected for the amount of administered drug and differences in the extraction efficiency of IS and ADR in the various tissues. Tissue levels were also corrected for ADR present in the blood in the tissue. Fig. $2 a$ and $b$ shows that the ADR levels in non-target tissues upon administration of ADR-AHCMS were lower than those after injection of free ADR. The ADR levels in the liver in the free drug group remained constant at approx. 15-20 $\mu \mathrm{g} / \mathrm{g}$ for $240 \mathrm{~min}$. In the ADR-AHCMS group, however, the initial ADR level was much higher $(37 \mu \mathrm{g} / \mathrm{g})$, and decreased to a level 4-times lower $(4.5 \mu \mathrm{g} / \mathrm{g})$ than that of the free drug group at $t=240 \mathrm{~min}$. The
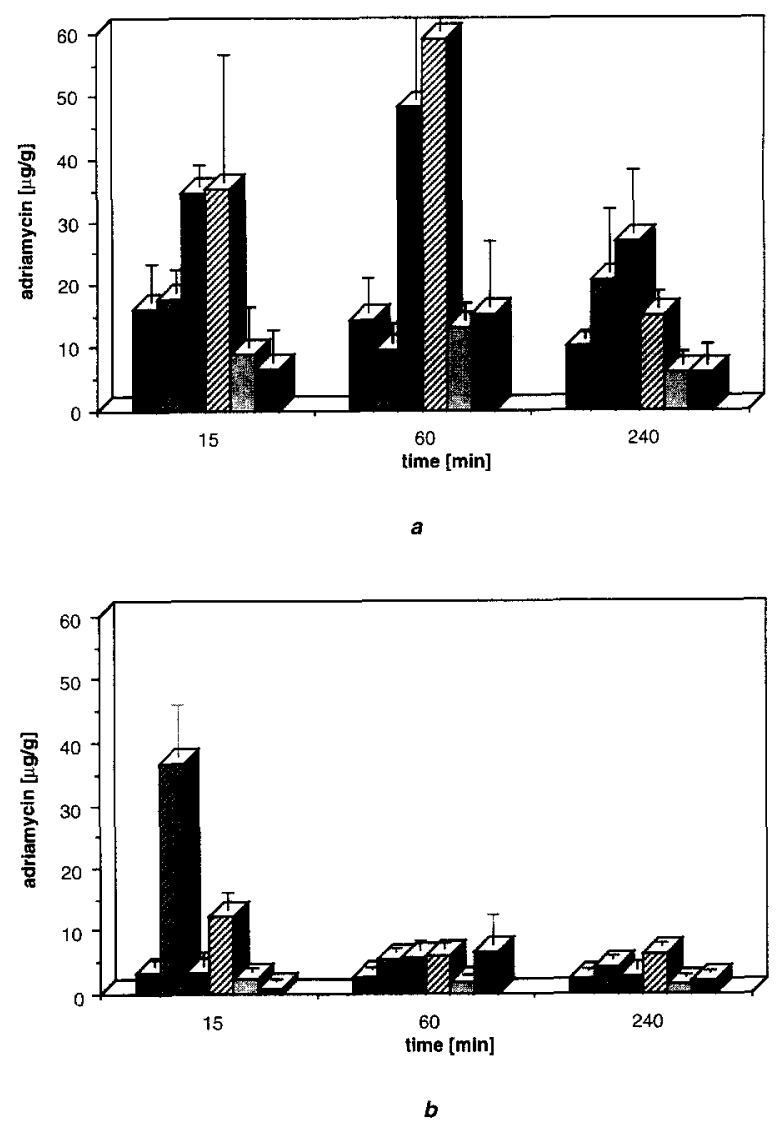

Fig. 2. ADR tissue levels at 15,60 and $240 \mathrm{~min}$ after i.v.p. administration of $5 \mathrm{mg} / \mathrm{kg} \mathrm{ADR}$ in male Wag/Rij rats. (a) Free ADR $\left(n_{15}=3, n_{60}=3, n_{240}=4\right)$; (b) ADR-AHCMS $(n=2)$. ( $\square)$ Lung; (田) liver; ( $(0)$ spleen; ( $(\nabla)$ kidney; ( $\square$ ) heart; ( $($ ) intestine. Error bars in (a) indicate SD; error bars in (b) denote range of observations.

larger decline of the ADR content in the liver may also be explained by the enhanced metabolism, due to increased bioreduction. It should be taken into account, however, that the initially higher drug content, measured in liver tissue after ADR-AHCMS administration, may be a consequence of ADR present in AHCMS lodged in the liver, which had not been released at that time. Nevertheless, whether the drug is present in the tissue itself or is still entrapped in microspheres in the tissue, it may be concluded that targeting of the drug to the liver using AHCMS is effective, since initially higher drug levels are achieved while minimising non-target 


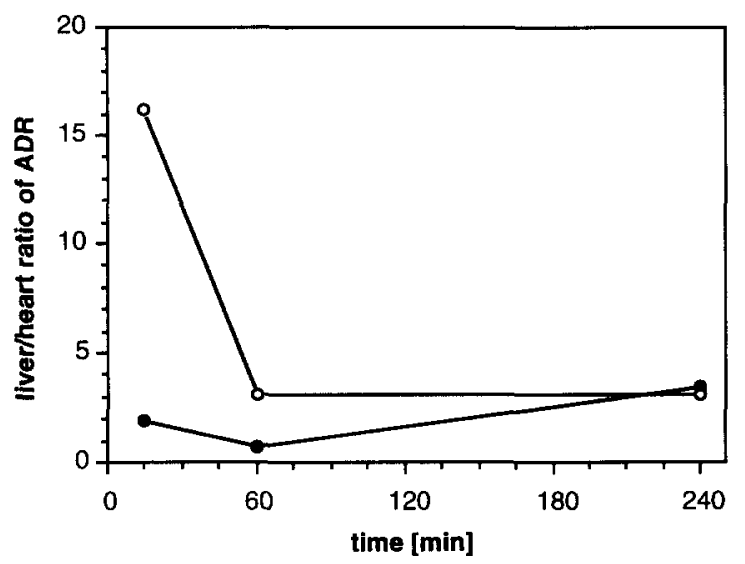

Fig. 3. Liver to heart ratio of ADR tissue levels after i.v.p. administration of $5 \mathrm{mg} \mathrm{ADR} / \mathrm{kg}$ to male $\mathrm{Wag} / \mathrm{Rij}$ rats. (•) Free ADR; O: ADR-AHCMS.

tissue levels. Moreover, the reduction of ADR heart levels which are approx. 5-times lower if the drug is administered in microspheres, is most important considering the cardiotoxicity of the drug. The increased targeting efficiency by formulation of the drug in AHCMS is thus demonstrated by the ratios of $A D R$ in liver and heart tissue, shown in Fig. 3. The increased liver to heart ratios, after administration of ADRAHCMS, indicate improved site-specific drug delivery.

\section{2. $A D R$ and $A D R-A H C M S$ cardiotoxicity in iso- lated working rat heart model}

General toxicity of ADR after i.v.p. administration as free drug or as microsphere-formulated drug was studied in $\mathrm{Wag} / \mathrm{Rij}$ rats. In the first group $(n=15)$, the rats were administered a dose of $7.5 \mathrm{mg} \mathrm{ADR} / \mathrm{kg}$, as a free drug. Due to high injection efficiency in the case of ADR-AHCMS, rats of the second group $(n=15)$ were administered $8.0 \mathrm{mg} \mathrm{ADR} / \mathrm{kg}$ as a microsphere formulation. The third group $(n=15)$ served as a control group and was not treated. Two animals, one in each treatment group, died within $24 \mathrm{~h}$ after injection. During the experiment, six more animals in the free drug treated group died. In both treatment groups the body weight of the animals decreased as compared to the control animals. In the case of the ADR-AHCMS treated animals there seemed to be some recovery from the initial toxicity, since these animals started to gain weight again from 2 weeks on, whereas the free drug treated group continued losing weight during the experiment. Differences between the three groups were significant $(p<0.05)$.

In the free drug treated group, the haematocrit and the haemoglobin content decreased immediately compared with the controls, whereas no differences were observed between the controls and the ADR-AHCMS treated group. The leucocyte count in the free ADR group at 1 week post-administration was only $30 \%$ of that of the controls. In the ADR-AHCMS treated group the leucocyte count was initially $70 \%$ of that of the controls, but was restored after approx. 4 weeks. This is in agreement with the results of Codde et al. (1990) which reported leucopenia following intra-arterial and intravenous administration of free ADR, whereas administration of ADR bound to sulphonated polystyrene microspheres did not significantly reduce the white blood cell count.

Already 1 week post treatment, measurements of haematological parameters (leucocyte count, haematocrit, haemoglobin, urea and creatinine content) became impossible in the free ADR treated group. Blood coagulated despite the heparin. The serum was also turbid, which was ascribed to hyperlipidaemia as could be shown by centrifugation of the serum. Bizzi et al. (1983) reported hyperlipidaemia in male CD-COBS rats 14-21 days after administration of a single dose of $\mathrm{ADR}(7.5 \mathrm{mg} / \mathrm{kg})$, resulting from a developing nephrotic syndrome. The rats in the free ADR treated group also had diarrhoea and ascites. Renal damage in this group was indicated by urea and creatinine levels which increased within 1 week, whereas the urea and creatinine levels in the control group and the ADR-AHCMS treated group remained at a constant level. 8 weeks after treatment, kidneys were removed and histological examination showed severe damage of the kidneys in the free ADR treated groups. In the kidneys of this group, vacuoles in the glomeruli, glomerular sclerosis, tubular dilatation, dense protein casts and interstitial fibrosis with cell infiltration were observed. No differences were 
observed between kidneys of the ADR-AHCMS treated group and the controls (data not shown). Nephrotic syndrome was probably the main cause of death in the animals in the free ADR treated group. This agrees with reports of Storm et al. (1989) who concluded that ADR-induced nephropathy represents an important cause of death in experimental animals.

The cardiotoxicity of ADR and ADR-AHCMS was evaluated using an isolated working heart model. In Table 1 several cardiac performance parameters, together with the body and heart weight of the animals in the different treatment groups are presented. Table 1 shows that no significant differences in the cardiac performance parameters were found. At histological examination, prominent histopathological differences between the three groups were absent. In both drug treated groups, however, some fibrillar loss was observed. Macroscopically, the hearts from the free drug treated group seemed somewhat flabby in comparison with hearts in the other groups and the left ventricular wall of these hearts was thinner than that of the two other groups.

Although heart weights were significantly decreased in ADR treated animals in both treatment groups and some heart damage was observed on a microscopic level in the free drug treated group, no significant changes in cardiac function were measured. It has been reported that morphological and histological changes do not always correlate with loss of cardiac function. Isner et al. (1983) found that $35 \%$ of patients with impaired left ventricular systolic perfor- mance had no light microscopic histological signs of toxicity, whereas in $52 \%$ of the patients without clinical toxicity, histological abnormalities were present. Reeves et al. (1990) suggested that the prediction and quantitation of ADR cardiotoxicity based on light microscopic changes is uncertain. An explanation for lack of functional impairment may be that both peak plasma levels and cumulative doses are important factors in the development of cardiac disfunction. Iatropolous (1984) reported that the earliest evidence for ADR associated cardiomyopathy was morphological, followed by clinical signs after two to five additional doses. Cummings and Smyth (1988) reported that ADR-induced cardiotoxicity has been more closely linked with repeated drug treatment. Yeung et al. (1989), however, studied changes in cardiac function in Sprague Dawley rats after a single intravenous ADR injection of $1-4 \mathrm{mg} / \mathrm{kg}$. There was an initial phase of rapid decline in cardiac output in the first 12 weeks and a second phase of persistent depression in cardiac function. These data suggest that strain differences may play an important role in the development of cardiomyopathy. This was also reported by Jaenke and Fajardo (1977) who found species and strain related variations in toxicity of anthracyclines in kidney and heart.

Since in rats the chronic toxicity of ADR not only includes cardiotoxicity but also nephrotoxicity, the question was raised whether nephrotoxicity adds to or even might be responsible for ADR-induced cardiotoxicity in rats, in fact it was suggested by Van der Vijgh et al. (1978) that

Table 1

Cardiac performance and body and heart weights ${ }^{a}$ of ADR-treated animals measured in an isolated working heart model at a left atrial filling pressure of $15 \mathrm{cmH}_{2} \mathrm{O}$ and at an aortic pressure of $90 \mathrm{cmH}_{2} \mathrm{O}$

\begin{tabular}{lccc}
\hline & Control $(n=12)$ & Free ADR $(n=6)$ & ADR-AHCMS $(n=12)$ \\
\hline Body weight $(\mathrm{g})$ & $302.0 \pm 5.5$ & $197.9 \pm 6.3^{\mathrm{b}, \mathrm{c}}$ & $261.9 \pm 8.6^{\mathrm{b}}$ \\
Heart weight $(\mathrm{g})$ & $1.2 \pm 0.0$ & $0.9 \pm 0.0^{\mathrm{b} . \mathrm{c}}$ & $1.1^{\mathrm{b}} \pm 0.1^{\mathrm{b}}$ \\
HW/BW & $0.4 \pm 0.0$ & $0.5 \pm 0.0$ & $0.4 \pm 0.0$ \\
Heart rate (beats $/ \mathrm{min})$ & $175.8 \pm 13.0$ & $148.7 \pm 11.2$ & $170.3 \pm 12.0$ \\
Coronary flow $(\mathrm{ml} / \mathrm{min})$ & $11.4 \pm 1.0$ & $8.6 \pm 0.5$ & $11.7 \pm 1.2$ \\
Cardiac output $(\mathrm{ml} / \mathrm{min})$ & $28.9 \pm 2.8$ & $24.5 \pm 3.2$ & $30.7 \pm 3.0$ \\
\hline
\end{tabular}

\footnotetext{
a Parameters are expressed as mean $\pm \mathrm{SE}$.

${ }^{b}$ Significantly different from controls, $p<0.05$.

${ }^{c}$ Significantly different from ADR-AHCMS treated group, $p<0.05$.
} 
cardiotoxicity of $\mathrm{ADR}$ in rats is partly related to concomitant nephrotoxicity. In our study, however, nephrotoxicity was evident without loss of cardiac function. In a study of Van Hoesel et al. (1986) it was concluded that cardiomyopathy observed in Lou/M rats is a simultaneous phenomenon independent of nephropathy.

From the initial decrease in body weights of treated animals, it can be concluded that, at an ADR dose of $7.5 \mathrm{mg} / \mathrm{kg}$, the drug is toxic to the animals. ADR-AHCMS treated animals started gaining weight again whereas free drug treated animals continued losing weight, demonstrating reduced toxicity of the microsphere formulated drug. The same trend was observed from the survival of the animals, seven animals in the free drug treated group died whereas in the ADRAHCMS group only one animal died. From the evaluation of the haematological parameters it was also concluded that the ADR-AHCMS was less toxic than free ADR. In the latter group leucocyte count, haemoglobin content and haematocrit was significantly decreased, whereas there was no difference between the ADRAHCMS treated animals and the controls, except for a transient small decrease in the leucocyte count, as far as these parameters are concerned. Nephrotoxicity was manifest in the free ADR treated group, while there was no change in renal function and kidney histology in ADR-AHCMS treated animals and controls. Overall, these results suggest that the toxicity of the drug is reduced when formulated in AHCMS.

\subsection{Dose tolerance of ADR-AHCMS after i.h.a. administration in rats}

Administration of ADR-AHCMS into the hepatic artery (i.h.a.) of Wag/Rij rats was more difficult than i.v.p. administration. The i.h.a. administration was carried out by injection into a small-diameter catheter. Injecting ADR solutions was no problem. Injection of ADR-loaded or empty AHCMS was more difficult, because the catheter was sometimes occluded by the microspheres and it was laborious to empty the syringe completely. This is reflected in Table 2 by the differences between the planned dose and the administered dose. In the case of empty AHCMS, $61-100 \%$ of the particles was injected, whereas in the case of ADR-AHCMS only $45-52 \%$ was injected. ADR-AHCMS tended to aggregate and to stick to the wall of the syringe, a problem which was not observed with empty AHCMS. Table 2 also shows that the administration of microspheres was not very reproducible, which is indicated by the standard deviation (SD) of the means.

Fig. 4 demonstrates the survival of individual animals as a function of the administered doses of ADR. Fig. 4 shows that administrations of

Table 2

Planned and injected doses of ADR, AHCMS and ADR-AHCMS administered i.h.a. to male Wag/Rij rats in dose tolerance experiment

\begin{tabular}{|c|c|c|c|c|}
\hline \multirow[t]{2}{*}{ Group } & \multicolumn{2}{|c|}{ Planned dose (mg/kg) } & \multicolumn{2}{|c|}{ Administered dose $(\mathrm{mg} / \mathrm{kg})^{a}$} \\
\hline & (ADR-)AHCMS & ADR & (ADR)-AHCMS & ADR \\
\hline$\overline{\mathrm{ADR}-1}$ & & 5.00 & & $6.08 \pm 0.46$ \\
\hline ADR-2 & & 7.50 & & $9.93 \pm 0.33$ \\
\hline ADR-3 & & 10.00 & & $11.88 \pm 1.45$ \\
\hline AHCMS-1 & 15.00 & & $12.44 \pm 1.36$ & \\
\hline AHCMS-2 & 22.50 & & $13.85 \pm 4.65$ & \\
\hline AHCMS-3 & 30.00 & & $20.32 \pm 6.71$ & \\
\hline AHCMS-4 & 45.00 & & $45.90 \pm 26.15$ & \\
\hline ADR-AHCMS-1 & 20.00 & 5.00 & $10.26 \pm 0.25$ & $2.68 \pm 0.07$ \\
\hline ADR-AHCMS-2 & 30.00 & 7.50 & $13.72 \pm 1.27$ & $3.58 \pm 0.33$ \\
\hline ADR-AHCMS-3 & 40.00 & 10.00 & $21.10 \pm 0.35$ & $5.51 \pm 0.09$ \\
\hline ADR-AHCMS-4 & 60.00 & 15.00 & $30.43 \pm 14.02$ & $7.94 \pm 3.66$ \\
\hline
\end{tabular}

\footnotetext{
${ }^{\mathrm{a}}$ Mean \pm SD.
} 


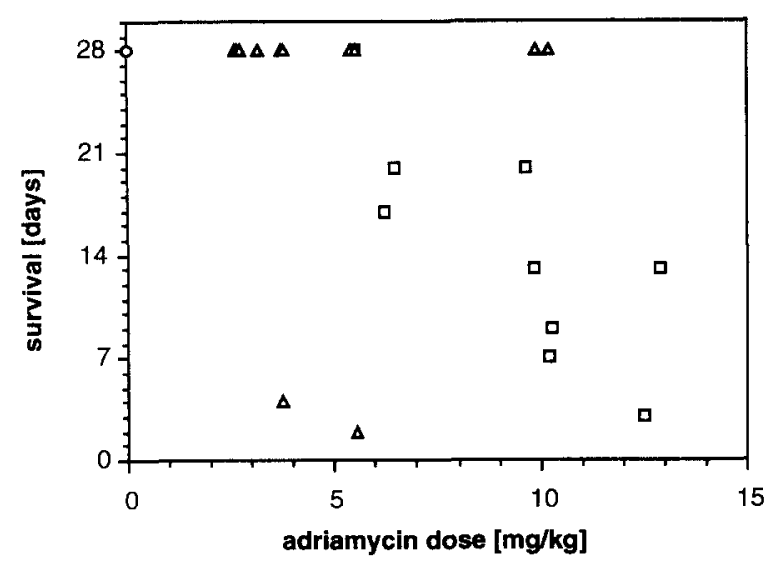

Fig. 4. Survival of maleWag/Rij rats after i.h.a. administration of ADR as a function of the drug dose. ( $\square$ ) Free ADR; $(\triangle)$ ADR-AHCMS; (O) control. Each point represents one animal.

ADR-AHCMS up to a level of $10 \mathrm{mg} \mathrm{ADR} / \mathrm{kg}$ are tolerated, even though two rats died at lower doses (cause unknown). For free drug there is a distinct decrease in survival times with increasing drug dose, although individual differences are obvious. From Fig. 4 the maximum allowable dose of free ADR is estimated to be approx. 4 $\mathrm{mg} / \mathrm{kg}$.

The differences in the dose tolerance of the ADR-formulations was also indicated by the changes of the average body weights of the animals in each group. The animals treated with the free drug lost weight, indicative of severe toxicity, with most animals dying within 20 days. One animal treated at the lowest dose of free ADR (ADR-1), survived and recovered, which was indicated by the weight gain of this animal. Animals treated with empty microspheres at any dose grew at the same rate as the control animals. The animals treated with the ADR-AHCMS initially lost weight, but after approx. 5 days the animals seemed to recover and gained weight again.

From the biochemical determination listed in Table 3 it can be seen that in the lowest free ADR group (ADR-1), there is a very mild change of the renal function, indicated by a small increase in urea levels, while creatinine levels are maintained at a normal level. In the $7.5 \mathrm{mg} / \mathrm{kg}$ group (ADR-2), there was only one animal alive at the time of the biochemical determination, and its values showed a greater change in the renal function. In most of the animals in the free drug group, there was a decrease in the alkaline phosphatase (AF) levels which is difficult to explain.

The biochemical determinations of most animals in the empty AHCMS groups, were normal at all doses, but sometimes a mild change of the renal function was detected, which could not be correlated with the dose. No changes in the hepatic function were observed in any AHCMS group. In the ADR-AHCMS groups there is a progressive deterioration in renal function which was correlated with the increase in dosage of ADRAHCMS. No alterations of the hepatic function were detected in this group, which is in agreement with the results previously reported by Cremers et al. (1994b).

Overall, survival, body weights, and kidney and liver parameters of Wag/Rij rats, after i.h.a. ad-

Table 3

Kidney and liver parameters of male Wag/Rij rats, 14 days after i.h.a. administration of ADR, empty AHCMS or ADRAHCMS

\begin{tabular}{|c|c|c|c|c|c|c|}
\hline Group $^{a}$ & $\begin{array}{l}\text { Creati- } \\
\text { nine }\end{array}$ & Urea & $\mathrm{g}-\mathrm{GT}$ & SGOT & SGPT & $\mathrm{AF}$ \\
\hline \multirow[t]{2}{*}{ Control } & 83 & 12.8 & 43 & 73 & 49 & 109 \\
\hline & 85 & 10.6 & 43 & 66 & 39 & 121 \\
\hline \multirow[t]{3}{*}{ ADR-1 } & 100 & 35 & - & - & - & - \\
\hline & 49 & 9.3 & 1 & 64 & 36 & 48 \\
\hline & 106 & 18.5 & 2 & 86 & 34 & 53 \\
\hline ADR-2 & 125 & 75.9 & 43 & 158 & 48 & 35 \\
\hline AHCMS-1 & 88 & 9 & 3 & 54 & 39 & 211 \\
\hline \multirow[t]{3}{*}{ AHCMS-2 } & 138 & 16.4 & 43 & 54 & 46 & 150 \\
\hline & 77 & 9.7 & 43 & 53 & 42 & 138 \\
\hline & 214 & 33 & 43 & 72 & 47 & 47 \\
\hline \multirow[t]{2}{*}{ AHCMS-3 } & 127 & 16.4 & 43 & 52 & 44 & 160 \\
\hline & 109 & 14.1 & 43 & 52 & 44 & 139 \\
\hline \multirow[t]{2}{*}{ AHCMS-4 } & 66 & 9.6 & 43 & 72 & 49 & 148 \\
\hline & 116 & 14.7 & 3 & 52 & 40 & 154 \\
\hline \multirow[t]{3}{*}{ ADR-AHCMS-1 } & 66 & 10.6 & 43 & 64 & 38 & 126 \\
\hline & 138 & 21.2 & 43 & 55 & 41 & 63 \\
\hline & 74 & 9.3 & 43 & 48 & 45 & 154 \\
\hline \multirow[t]{2}{*}{ ADR-AHCMS-2 } & 83 & 11.8 & 43 & 52 & 42 & 139 \\
\hline & 118 & 15.6 & 43 & 51 & 45 & 159 \\
\hline \multirow[t]{2}{*}{ ADR-AHCMS-3 } & 153 & 19 & 3 & 51 & 49 & 148 \\
\hline & 155 & 19.3 & 5 & 59 & 38 & 218 \\
\hline \multirow[t]{2}{*}{ ADR-AHCMS-4 } & 144 & 14.8 & 3 & 47 & 41 & 140 \\
\hline & 282 & 27.6 & 9 & 55 & 38 & 135 \\
\hline
\end{tabular}

${ }^{\text {a }}$ Codes are explained in Table 2. 
ministration of free ADR, AHCMS and ADRAHCMS suggest that the adriamycin toxicity is reduced if the drug is formulated in AHCMS. At higher dosages, ADR-AHCMS are better tolerated than free ADR. Intrahepato-arterial administration of empty AHCMS was tolerated at any dose tested. From these results it is concluded that in the case of i.h.a. administration in Wag/Rij rats, free ADR is tolerated at a dose of $4 \mathrm{mg} / \mathrm{kg}$, whereas in the case of ADR-AHCMS, a dose of $10 \mathrm{mg} \mathrm{ADR} / \mathrm{kg}$ could be administered.

\section{Conclusions}

Albumin-heparin conjugate microspheres loaded with adriamycin can be used for sitespecific delivery of the drug. After intrahepatic administration of the drug by i.v.p. injection, adriamycin peak plasma concentrations and nontarget tissue levels were significantly reduced, whereas drug levels in the target tissue were initially increased, if the drug was formulated in AHCMS (as compared with administration of free drug). Adriamycin cardiotoxicity could not be determined using cardiac performance parameters, since these parameters were influenced neither by free drug nor by ADR-AHCMS at the given dose and time scale. Acute toxicity after i.v.p. administration as determined by body weight, leucocyte count, haematocrit, and haemoglobin, urea and creatinine levels, however, demonstrated that at the same drug dose ADR-AHCMS is tolerated better than free ADR.

Administration of ADR, AHCMS and ADRAHCMS into the hepatic artery also demonstrated that the microsphere formulated drug is less toxic than free ADR. At the same drug dose, survival of animals is increased, and animals recover from initial weight loss due to acute toxicity if the drug is administered as ADR-AHCMS instead of free ADR. Nephrotoxicity increased with increasing drug dosages with higher doses being tolerated if the drug is formulated in AHCMS as compared to free ADR. Survival, changes in body weight and biochemical parameters of animals treated with empty AHCMS did not markedly differ from the controls, which only received a sham operation indicating that the empty microspheres are not toxic to the liver tissue.

\section{Acknowledgements}

Thanks are due to the Netherlands Organization for Scientific Research (NWO) and Theratech Inc., Salt Lake City, UT, for financially supporting this research and Pharmachemie, Haarlem, The Netherlands for the kind gift of adriamycin. The assistance of $L$. Houtdijk with the operations during the intra-arterial administration experiments is greatly acknowledged.

\section{References}

Bielack, S.S., Erttmann, R., Winkler, K. and Landbeck, G., Doxorubicin: Effect of different schedules on toxicity and anti-tumor efficacy. Eur. J. Cancer Clin. Oncol., 25 (1989) 873-882.

Bizzi, A., Ceriani, L., Gerundino, M. Spina, A.M., Tacconi, M.T. and Veneroni, E., Adriamycin causes hyperlipidemia as a consequence of nephrotoxicity. Toxicol. Lett., 18 (1983) 291-300.

Camaggi, C.M., Comparsi, R., Strocchi, E., Testoni, F., Angelelli, B. and Pannuti, F., Epirubicin and doxorubicin comparative metabolism and pharmacokinetics. Cancer Chemother. Pharmacol., 21 (1988) 221-228.

Codde, J.P., Burton, M., Kelleher, D.K., Archer, S.G. and Gray, B.N., Reduced toxicity of adriamycin by incorporation into ion exchange microspheres: A therapeutic study using a rat liver tumour model. Anticancer Res., 10 (1990) 1715-1718.

Cremers, H.F.M., Kwon, G.S., Bae, Y.H., Kim, S.W., Verrijk, R., Noteborn, H.P.J.M. and Feijen J., Preparation and characterization of albumin-heparin microspheres. Biomaterials, 1 (1994a) 38-48.

Cremers, H.F.M., Lam, K., Wolf, R.F.E., Blaauw, E.H., Schakenraad, J.M., Nieuwenhuis, P., Verrijk, R., Kwon, G.S., Bae, Y.H., Kim, S.W. and Feijen J., Degradation and intrahepatic compatibility of albumin-heparin conjugate microspheres. Biomaterials, 15 (1994b) 941-947.

Cremers, H.F.M., Verrijk, R., Noteborn, H.P.J.M., Kwon, G.S., Bae, Y.H., Kim, S.W. and Feijen J., Adriamycin loading and release characteristics of albumin-heparin conjugate microspheres. J. Controlled Rel., 29 (1994c) 143155.

Cummings, J. and Smyth, J.F., Pharmacology of adriamycin: The message to the clinician. Eur. J. Cancer Clin. Oncol., 24 (1988) 579-582. 
Iatropolous, L.F., Anthracycline cardiomyopathy: Predictive value of animal models. Cancer Treat. Symp., 3 (1984) 3-17.

Isner, J.M., Ferrans, V.J., Cohen, S.R., Witkind, B.G., Virmani, R., Gottdiener, J.S., Beck, J.R. and Roberts, W.C., Clinical and morphologic cardiac findings after anthracycline chemotherapy: analysis of 64 patients studied at necropsy. Am. J. Cardiol., 51 (1983) 1167-1174.

Jaenke, R.S. and Fajardo, L.F., Adriamycin-induced myocardial lesions: report of a workshop. Am. J. Surg. Pathol., 1 (1977) 55-651

Reeves, W.C., Griffith, J.W., Wood, M.A. and Whitesell, L., Exacerbation of doxorubicin cardiotoxicity by digoxin administration in an experimental rabbit model. Int. J. Cancer, 45 (1990) 731-736.

Storm, G., Van Hoesel, Q.G.C.M., De Groot, G., Kop, W., Steerenberg, P.A. and Hilen, F.C., A comparative study on the antitumor effect, cardiotoxicity and nephrotoxicity of doxorubicin given as a bolus, continuous infusion or entrapped in liposomes in the Lou/M Wsl rat. Cancer Chemother. Pharmacol., 24 (1989) 341-348.

Van der Vijgh, W.J.F., Van Velzen, D., Van der Poort, J.S.E.M., Schlüper, H.M.M., Mross, K., Feijen, J. and
Pinedo, H.M., Morphometric study of myocardial changes during puromycin aminonucleoside induced nephropathy in rats. Anticancer Res., 7 (1978) 1111-1116.

Van Hoesel, Q.G.C.M., Steerenberg, P.A., Dormans, J.A.M.A., De Jong, W.H., De Wildt, D.J. and Vos, J.G., Time-course study on doxorubicin-induced nephropathy and cardiomyopathy in male and female Lou/M Wsl rats: Lack of evidence for a causal relationship. J. Natl. Cancer Inst., 76 (1986) 299-307.

Willmott, N., Cummings, J., Marley, E. and Smyth, J.F., Relationship between reductive drug metabolism in tumour tissue of anthracyclines in microspherical form and anti-tumour activity. Biochem. Pharmacol., 39 (1990) 1055-1062.

Wondergem, J., Van der Laarse, A., Van Ravels, F.J.M., Van Wermeskerken, A.M., Verhoeve, H.R., De Graaf, B.W. and Leer, J.W., In vitro assessment of cardiac performance using an isolated working rat heart preparation. Int. J. Radiat. Biol., 59 (1991) 1053-1068.

Yeung, T.K., Simmonds, R.H. and Hopewell, J.W., A functional assessment of the relative cardiotoxicity of adriamycin and epirubicin in the rat. Radiother. Oncol., 15 (1989) 275-284. 